\title{
Processing of geometrical dimensions in a binary classification task: Evidence for a dual process model
}

\author{
P. J. G. KEUSS \\ Free University, Amsterdam, The Netherlands
}

\begin{abstract}
Confirming the findings in search tasks with letters and digits, the typical $\mathrm{RT}_{\text {same }}<\mathrm{RT}_{\text {diff }}$ result was obtained in a matching paradigm requiring the classification of geometrical stimuli that were given in pairs. The study supports a dual process model that is based on an identity reporter for the faster "same" response and a difference detector for the slower "different" responses, both operating with equal accuracy. Subjects appeared to perceive outline aspects of figures, formed by size and form, holistically. An internal characteristic, such as an interior line, was apparently processed as a separate attribute. However, the outlines of the stimulus configurations appeared to be much more salient and interfered with the judgment of the orientation of the interior line. Moreover, the latter stimulus aspect could be easily ignored as the task required.
\end{abstract}

Following the work of Egeth (1966), several other investigators have used his paradigm to study the mode of processing of simple geometrical stimuli (Grill, 1971; Hawkins, 1969; Nickerson, 1967, 1972). In this research, pairs of stimuli composed of combinations of varying dimensions, such as size, form, and color of the figures or the orientation of an inscribed line, are presented and the subjects' task is to judge as quickly as possible whether or not the pairs are identical.

Originally, it was supposed that, in making his decision, the subject checked the various dimensions individually. If such a strategy is applied, the judgment time should be longest when all dimensions relevant to the "same" vs. "different" decision have to be analyzed. This situation evidently occurs when there are actually no differences on any of the dimensions and the subject has ultimately to conclude that the pairs are the same (Snodgrass, 1972).

Many of the results, however, were consistently at variance with the assumption of such an exclusively analytical model (cf. Nickerson's, 1972, survey). The "same" responses usually appeared to be too short. This confirmed RT outcomes from earlier investigations on comparison processes using alphanumeric stimuli, where several dual process models were proposed to interpret the $\mathrm{RT}_{\text {same }}<\mathrm{RT}_{\text {diff }}$ result (Bamber, 1969; Beller, 1970; Krueger, 1970; Tversky,

The author's address: Department of Psychology, Free University, Provisorium, De Boelelaan 1115, Amsterdam, The Netherlands. The research was partly supported by a grant from the Netherlands Organisation for the Advancement of Pure Research (Z.W.O.), The author is indepted to P. G. Pelle, H. H. Stenson, and A. Ortony, who made very helpful comments on an earlier draft of the paper, and to L. C. Boer for his able assistance in the collection and analysis of data.
1969; Marcel, Note 1). In most of these models, two different processors are distinguished: (1) an identity reporter based on a fast holistic match that is responsible for the faster "same" responses, and (2) a slower feature-by-feature analyzer for the "different"' responses.

It could be argued that, in the case of alphanumeric stimuli, the subject's ability to name the stimuli could account for the quicker "same" response (cf. Nickerson, 1975) in that he accesses only one name. Nevertheless, the main aim of the present study is to demonstrate the need to postulate such an identity reporter for the judgment of geometrical stimuli which are not readily nameable. Supposedly, matches in such a discrimination task are primarily based on visual characteristics. Then, some global impression of similarity is presumed to be responsible for the "same" response, viz, the identity reporter is operating on the visual representation of patterns instead of the individual dimensions that constitute the figures.

First, however, it should be noted that in binary classification tasks following Egeth (1966), the relative brevity of the "same" response can be relatively easily produced without conclusively establishing a fast identity reporter. (For extensive criticisms on procedural shortcomings, see Grill, 1971; Krueger, 1973; Silverman \& Goldberg, 1975.) In short, when the figures to be compared are allowed to vary on one or more dimensions, the "different" response is appropriate to a variety of figure pairs being different on one, some, or all dimensions. In contrast, the "same" response is required only to those few figure pairs which are completely identical. This implies that all "same"' responses are determined by homogeneity, whereas only the presence of a maximal 
difference between the figures of a pair would determine a unanimous or homogeneous "different" response. Furthermore, the "same" responding system, being related to a smaller number of alternatives (or even one!), may be "primed," as Silverman and Goldberg (1975) noticed. An additional procedural artifact leading to faster "same" reactions is the higher proportion of stimulus repetitions for identicals required maintaining equal probabilities for correct "same" and "different" responses (cf. Felfoldy, 1974; Krueger, 1973). Hence, for the determination of an identity reporter, it would be more adequate to compare "same" responses against those "different" responses that are considered to be homogeneous. And, in line with the Silverman and Goldberg (1975) study, we consider maximally differing stimuli to be the logical inverse of completely identical stimuli. Further, it is clear that the set size of the stimuli corresponding to either type of response must be equal.

In criticizing Egeth's (1966) paradigm, Well (i $\ni 71$ ) pointed to another source of ambiguity in RT outcomes in binary classification tasks. This was that prior relevance of irrelevant dimensions may interfere with the act of attending to the relevant dimensions. Therefore, in the present study, confounding effects due to the subjective importance attributed to particular, though not relevant, dimensions and confusion between relevant and irrelevant dimensions was eliminated by instructing the subject to attend only to one dimension during the experiment. This means that he had to base his "same" or "different" response on that dimension alone.

A second aim of the study was to answer the empirical question of whether the activity of the identity reporter is contingent upon total congruence of the stimulus pairs or whether its activity can be directed towards the dimension of relevance. For that purpose, the figures were composed of two dimensions, the relevant one that varied from trial to trial and an irrelevant one that could be ignored or filtered out by the subject. Further, in contrast to the relevant one, the irrelevant dimension was kept in a fixed state that could be either "same" or "different," thereby also reducing confusion between the relevant and irrelevant dimension.

In demonstrating the selective activity of the identity reporter, it is of interest to examine whether the $\mathrm{RT}_{\text {same }}<\mathrm{RT}_{\text {diff }}$ result can be obtained while the irrelevant dimension actually produces a difference between the figures. It is clear that, in that case, the act of ignoring or neglecting irrelevant stimulus aspects may be helpful for the identity reporter. Or, to put it in analytical terms, the "same" responding system suffers from "state" incompatibility (Hawkins, McDonald, \& Cox, 1973), since the irrelevant dimension calls for the opposite response to the relevant dimension. In contrast, when both relevant and irrelevant dimensions produce differences between the figures, the "different" responding system may in that case take advantage of "state" compatibility as both dimensions call for the "different" response. One has to keep in mind, however, that as the dissimilarity between the figures is maximal and as "same" as well as the "different" responses are required during the same test, the subject's tendency to give the "same" responses by default might be evoked, leading to the opposite result, namely $R T_{\text {same }}>R T_{\text {diff. }}$. To prevent the subject from adopting such a strategy, Hawkins' (1969) procedure is used, whereby selective responses to the stimuli are required so that the "same" and "different" responding systems are not placed in direct competition during the test. Therefore, in all conditions of the experiment, the stimuli were administered twice to the subjects. In one cycle, "same" responses were demanded when there was no difference in the relevant dimension, and in the second cycle, the "different" response was demanded when there was a difference in the relevant dimension.

In spite of the use of a filtering task in which one dimension is designated to be relevant and the other not, perceptual fusion of dimensions may be unavoidable. Some dimensions normally employed in this sort of task, such as form, size, and color, for instance, cannot be taken away or added at will, as Grill (1971) and Saraga and Shallice (1973) showed. Their empirical findings are in accord with the theoretical notions of Lockhead (1972) about stimulus integrality. Moreover, there are geometrical dimensions that may generate new nominally related stimuli, perceived as wholes, as Pomerantz and Garner (1973) found in card-sorting tasks. They concluded that these perceptual phenomena may be due to configural properties.

Hence, it also seemed useful to present the dimensions here employed (size and form of the figures and the orientation of an interior line) alone to yield reference RTs against which to compare the speed of processing of the multidimensional stimuli. Further, we can investigate whether the identity reporter, based on template or "gestalt" matching, may be prompted by integral stimuli. The one might expect a much greater advantage of the "same" responses over the "different" ones in comparison with nonintegral stimuli.

\section{METHOD}

\section{Subjects}

Twenty-four college freshmen at the Free University served as subjects. They fulfilled a course requirement, but also were paid a fixed sum for participation. All had normal or corrected-tonormal vision and used their preferred hands for responding. Eight of them were assigned at random to each part of the experiment. 


\begin{abstract}
Apparatus
A PDP 8-I computer controlled the presentation and duration of the stimuli, time intervals, and counting and recording of elapsed judgment times. The kw 8-IE real-time clock with a basic frequency of $1,000 \mathrm{~Hz}$ was started by the onset of the stimulus and halted upon keypressing. There was a single response key connected to a microswitch that required a pressure of $5 \mathrm{oz}$ to be closed. The stimuli appeared on a $16 \times 21 \mathrm{~cm}$ Tectronix display unit (611). In a dimly lit room, the subject was permitted to sit in a comfortable position with his eyes about $50 \mathrm{~cm}$ from the screen.
\end{abstract}

\section{Materials}

The stimuli were pairs of simple geometrical figures. Each figure was positioned on either side of the center of the screen, with an interspace of $36 \mathrm{~mm}$ between their midpoints, which were situated on fixed coordinate matrices. The stimuli were presented simultaneously with rise and decay times within a 7 -msec limit. Stimulus duration was fixed at $1 \mathrm{sec}$, which proved to exceed the subjects reaction times in virtually all cases. The stimuli were composed of the dimensions of size (S), form (F), or orientation of an interior line $(\mathrm{O})$. Each dimension had two values. These values were selected in such a way that, by and large, discrimination time to each dimension was equal.

The experiment consisted of three parts, in each of which only one dimension, $\mathrm{S}, \mathrm{F}$, or $\mathrm{O}$, varied from trial to trial. In the experimental conditions, a second, irrelevant dimension selected from the remaining two was added to the relevant dimension. Its value was kept fixed during a stimulus series. With one irrelevant, but two-valued dimension, four sets of stimulus pairs and, hence, four different stimulus series with respect to that irrelevant dimension can be constructed. For instance, stimulus pairs formed by a combination of relevant and varying $O$ and irrelevant and constant $\mathbf{S}$ were presented in: two small, identical squares (first series); two large, identical squares (second series); left square small, right one large (third series); and left square large, right one small (fourth series). In an analogous way, with two irrelevant dimensions, eight different stimulus series per part were formed. The construction and measurements of all stimuli of the three parts is given in Table 1. Table 1 illustrates that each part contained a control condition with only the relevant dimension. Therefore, in Part $I$, pairs of squares were given measuring either $11 \times 11 \mathrm{~mm}$ or $16 \times 16 \mathrm{~mm}$. In Part 2, pairs of rectangles, both measuring $11 \times 16 \mathrm{~mm}$, were presented. For each figure, the longer side was either the height or the base $(h>b$ vs. $b>h)$. In part 3, pairs of lines were given. Each was $13.5 \mathrm{~mm}$ long, and its orientation was either horizontal or vertical.

The bidimensional stimuli of the experimental conditions were constructed by means of superposing the appropriate dimensions, the constraints being: (1) for stimuli involving 0 , interior lines were given, bisecting each of the figures of the stimulus; and (2) for stimuli involving both $S$ and $F$, two rectangles were used, measuring either $9 \times 13 \mathrm{~mm}$ or $13 \times 19 \mathrm{~mm}$. These areas approximate those of the squares and rectangles given under the control conditions of Parts 1 and 2, respectively.

\section{Design and Procedure}

Each part had 10 series of $\mathbf{3 0}$ trials. The stimuli of the first and last series contained only the relevant dimension, yielding con-

Table 1

Construction and Measurements of the Stimuli in the Three Parts

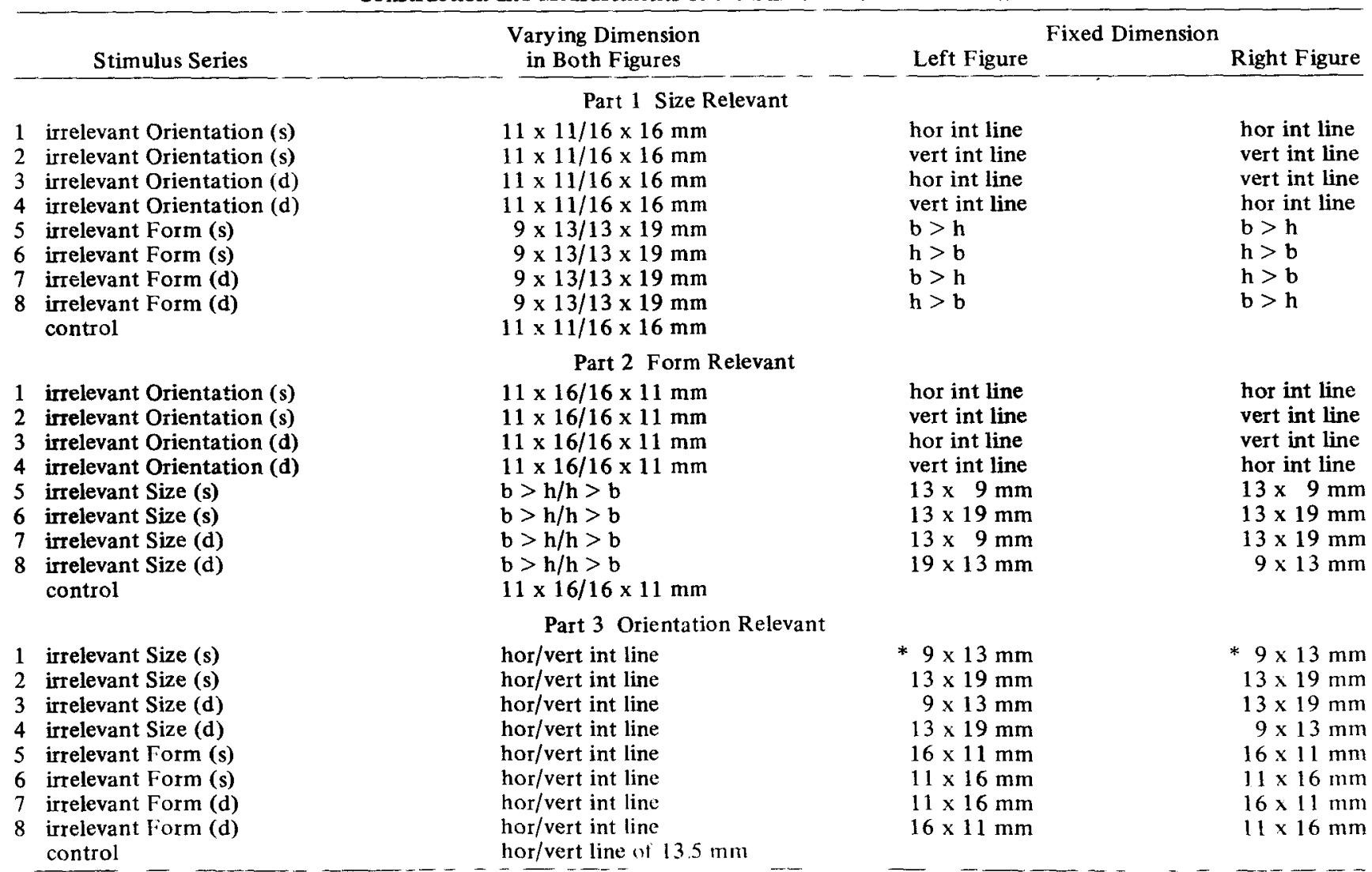

Note $-b=$ base, $h=$ height, hor $=$ horizontal, vert = lertical, $m$, minm, s = same $d=$ different.

*The first digit refers to the length of the base" 
trol data. Administration of the eight remaining series was counterbalanced across subjects.

Each subject was told to pay attention only to the varying dimension which was designated to be relevant to his "same" vs. "different" response. The experiment was run twice for each subject, using two identical cycles. In the one cycle, the subjects were instructed to respond by pressing the key, when the relevant dimension was different in the figures. In the other cycle, he was to respond when the relevant dimension did not make a difference. Half of the subjects started with the "same" responding requirement, and half of the subjects started with the "different" responding requirement.

The computer was programmed to select the stimuli at random from the four sets of stimulus pairs that can be formed by the value of the relevant dimension, the constraint being that for each subject the proportions of correct "same" and "different" responses were kept equal in each stimulus series. Equal emphasis was laid upon speed and accuracy.

A trial started with a yellowish-green light of $40 \mathrm{msec}$ flashing on the screen." This served as a "ready" signal. One second later, the stimulus appeared. Intertrial time was $3 \mathrm{sec}$. Erroneous responses resulted in an intertrial time prolongation of $2 \mathrm{sec}$. During that time, the computer provided feedback on the screen. Following an error, two extra trials chosen at random from the running stimulus series were added, but they were not used for the analysis. The error trials were repeated later on in the series.

The subject began with a practice session in order to become familiarized with the dimensions on which he had to base his response. Prior to each stimulus series, he was told which irrelevant dimension was added to the relevant one and 10 extra trials served for exercise. Between each stimulus series, there was a 5-min rest break. After each set of five series, this was lengthened to $30 \mathrm{~min}$. Total time required for each cycle was less than $2 \mathrm{~h}$, including the pauses. Each subject completed his two cycles, one on each of two consecutive days.

\section{RESULTS}

Mean $\mathrm{RT}_{\text {same }}$ and $\mathrm{RT}_{\text {diff }}$ to the relevant dimension of each part are plotted in the upper sections of Figure 1, where the data of each part are brought together under three conditions that are determined by the type and state of the irrelevant dimension. Under $s$ and $d$ conditions, the irrelevant dimension was in "same" and "different" state, respectively. For clarity, the data of the control (o) conditions, which omitted the irrelevant dimension, are plotted twice in each part as reference for the addition of each of the irrelevant dimensions that was added to the relevant one.

The ANOVA on all RT data (with subjects as a nested factor) revealed the highest proportion of the variance to be attributable to type of response $[F(1,21)=47.2, p<.01]$. Figure 1 illustrates that in all three parts $\mathbf{R T}_{\text {same }}$ was considerably faster than $\mathrm{RT}_{\text {diff }}$.

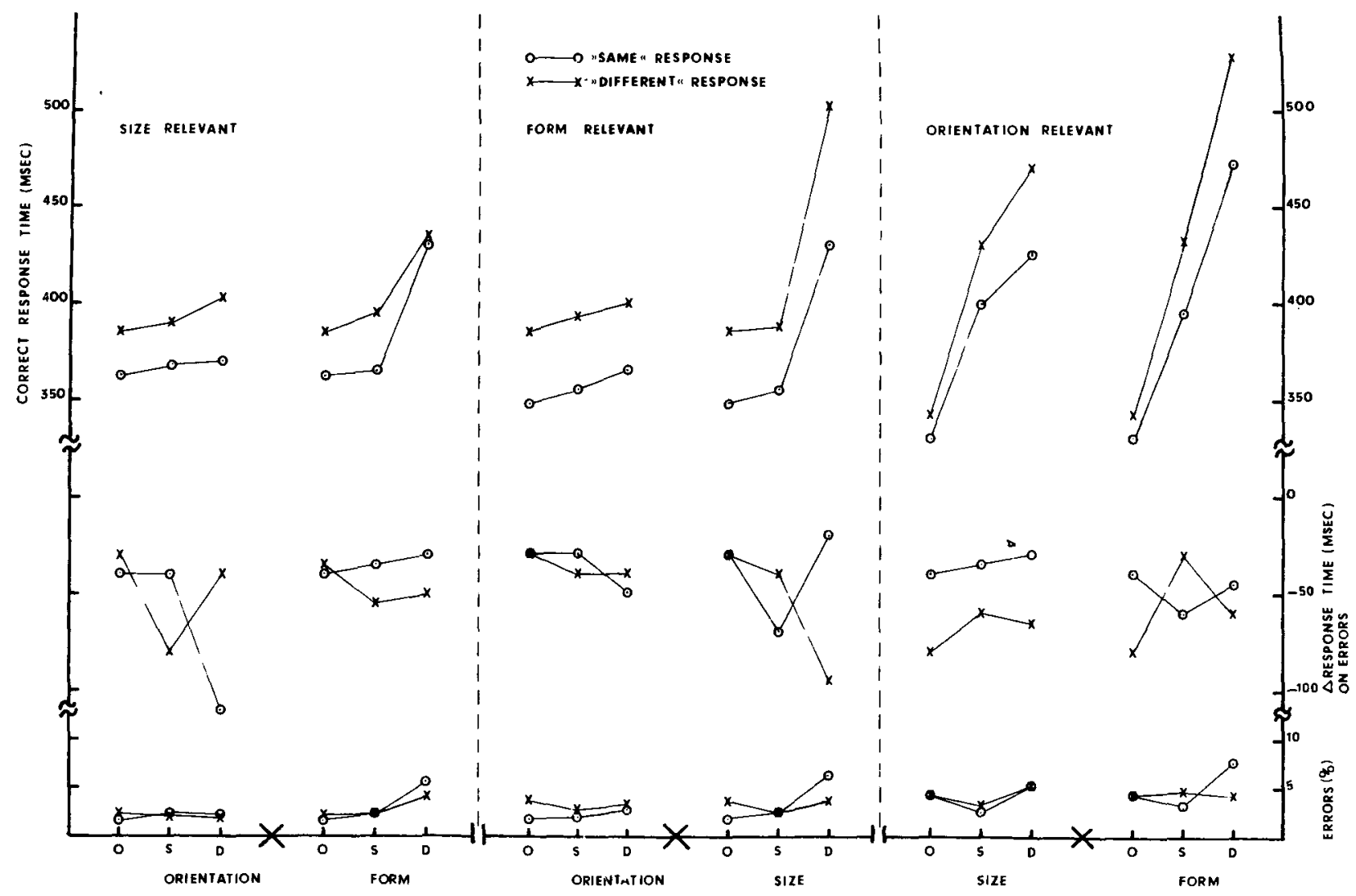

Figure 1. Mean "same" and "different" RTs, error frequencies, and mean RT differences between correct and erroneous responses as a function of the irrelevant dimension being in "same" (s) or "different" (d) state. The data of each part are separated by vertical dotted lines. The results of the (o) condition (controls) yielding reference points for each of both irrelevant dimensions are iloun $n$ iwice for each part. 
Type of relevant dimension fell short of the .05 level of significance, but showed a significant interaction with conditions $[F(4,21)=6.2, p<.01]$. In a second ANOVA, which omitted the control data, type of relevant dimension appeared to be a significant source of variance, and subsequent application of Duncan range test revealed a significant $R T$ advantage of size as well as of form over orientation $(\mathrm{p}<.05)$. Figure 1 clearly shows that the $\mathrm{RT}_{\text {same }}$ $<\mathrm{RT}_{\text {diff }}$ result was also obtained when the irrelevant dimension had a "different" state as under the $\mathrm{d}$ conditions. However, both "same" and "different" responses were significantly lengthened compared with those obtained under the s conditions, where the irrelevant dimension had a "same" state $[F(1,21)$ $=121.9, \mathrm{p}<.01$, in the second ANOVA]. The left and middle panel of Figure 1 show clearly that RT to relevant size and form is scarcely affected by the addition of an interior line (orientation). In contrast, RT to relevant orientation is considerably increased by the addition of irrelevant size or form.

\section{Errors}

For a total of 14,400 scores, the numbers of false "same" and "different" responses were 230 and 229 , respectively. All erroneous responses were given 20 to $120 \mathrm{msec}$ faster than the correct ones, while, on the average, the false "different" RTs were about 10 msec shorter than the false "same" RTs (see the middle sections of Figure 1). There is a slight, but statistically unreliable, trend for false "same" responses to occur more frequently when the state of the irrelevant dimension is "different" rather than "same."

\section{DISCUSSION}

The $\mathbf{R T}_{\text {same }}<\mathbf{R} \mathrm{T}_{\text {diff }}$ result obtained in all three parts strongly supports the hypothesis that a separate and fast identity reporter is operating. This process, therefore, does not seem to be restricted to search tasks with letters, digits, or other overlearned stimulus material. In the present study, its working was clearly demonstrated in the judgment process of geometrical stimuli that are not so easily nameable. Apparently, there is no loss in accuracy when compared with the working of the slower feature-byfeature process that presumably underlies the "different" responses: The error percentage for false "same" and false "different" responses was nearly equal. These findings together lead to the conclusion that a fast, global, and rough impression of similarity is sufficient for the match of such simple figures as employed in the present study, where nearly all judgments could take place within the duration of $1 / 2 \mathrm{sec}$. This indicates that the perceptual distance between similarity and dissimilarity was relatively large. Furthermore, the dimensions that constituted the figures were two-valued, and this implies that the number of ways in which the figures were allowed to differ was very restricted.

The $\mathrm{RT}_{\text {same }}<\mathrm{RT}_{\text {diff }}$ result was even found when the irrelevant dimension was in a different state, but in that case both response types appeared to be retarded compared to the situation when the irrelevant dimension was the same. Concerning the increase in $\mathrm{RT}_{\text {same, }}$ it seems reasonable to suppose that it was at the response execution stage and, thus, after the operation of the fast identity reporter, that "state" incompatibility affected the response time (cf. Hawkins et al., 1973). Yet the $\mathrm{RT}_{\text {diff }}$ was more time consuming in spite of the "state" compatibility of relevant and irrelevant dimension, which requires an explanation that is directed towards stimulus processing instead of response interference. When both dimensions, the relevant one and the irrelevant one, do differ in the figure pairs, the subject may encounter difficulties in determining on which dimension he must base his correct "different" response. Especially figures composed of one or two integral dimensions (size and form) may hamper the analysis of the figures into parts which may be typical for the difference detector, as it supposedly works on the basis of feature-by-feature testing.

Considering integrality of stimulus aspects, the data indicate that, in particular, the outlines of the stimulus configurations, as produced by size and form, were considered together. In fact, it appeared that internal characteristics as produced by orientation of the interior line could be neglected or ignored by the subject. The whole judgment process, regardless of the "same" vs. "different" outcome, was scarcely affected by the interior line and its orientation. Moreover, size and form seemed to be very salient, insofar as the outline aspects of the figures strongly interfered with the judgment of the orientation of the inserted line. In terms of dimensional separability (Pomerantz \& Garner, 1973), the outline aspects of the stimulus configurations at first glance tended to be perceived as a whole, whereas the internal aspects were conceived as a separate attribute. Further, the subject had a tendency to look, rather, at outline aspects or surroundings, even when irrelevant, than at internal or more "hidden" aspects.

A last question concerns the status of the separate difference detector, being responsible for the slower "different" reactions. The data might suggest that the subject could adopt a relatively simple stimulus matching strategy. After a mismatch, being the negative outcome of the fast identity reporter, he could provide the "different" response by default. Following this reasoning, however, a constant time added to the "same" RTs ought to fit the different RTs which is clearly at variance with the obtained RT data. Moreover, such a matching strategy would 
be inadequate in those situations when the irrelevant dimension was in a different state. Then the subject was forced to check each dimension in order to determine whether or not it contributed to the relevant difference between the figure pairs on which he had to base his response.

\section{REFERENCE NOTE}

1. Marcel, A. J. Serial and parallel processing in pattern recognition. Paper presented at the meeting of the Experimental Psychology Society, London, January 1969.

\section{REFERENCES}

BAMBER, D. Reaction times and error rates for "same"-."different" judgments of multidimensional stimuli. Perception \& Psycho. physics, 1969, 6, 169-174.

BELLER, H. K. Parallel and serial stages in matching. Journal of Experimental Psychology, 1970, 84, 213-219.

EGETH, H. E. Parallel versus serial processes in multidimensional stimulus discrimination. Perception \& Psychophysics, 1966, 1, 245-252.

Felfoldy, G. L. Repetition effects in choice reaction time to multidimensional stimuli. Perception \& Psychophysics, 1974, 15, 453-459.

Grill, D. P. Variables influencing the mode of processing of complex stimuli. Perception \& Psychophysics, 1971, 10, 51-57.

Hawkins, H. L. Parallel processing in complex visual discrimination. Perception \& Psychophysics, 1969, 5, 56-64.

Hawkins, H. L., McDonald, G. J., \& Cox, A. K. Effects of irrelevant information in speeded discrimination. Journal of Experimental Psychology, 1973, 98, 435-437.

KRUEGER, L. E. Effect of bracketing lines on speed of 'same''different' judgment of two adjacent letters. Journal of Experimental Psychology, 1970, 84, 324-330.

KRUEGER, L. E. Effects of stimulus frequency on speed of 'same''different' judgments. In S. Kornblum (Ed.), Attention and performance IV. New York: Academic Press, 1973. Pp. 497-506.
Lockhead, G. R. Processing dimensional stimuli: A note. Psychological Review, 1972, 79, 410-419.

Nickerson, R. S. Categorization time with categories defined by disjunctions and conjunctions of stimulus attributes. Joumal of Experimental Psychology, 1967, 73, 211-219.

Nickerson, R. S. Binary-classification reaction time: A review of some studies of human information-processing capabilities. Psychonomic Monograph Supplements, 1972, 4(17, Whole No. 65).

Nickerson, R. S. Effects of correlated and uncorrelated noise on visual pattern matching. In P. M. A. Rabbit \& S. Dornic (Eds.), Attention and performance V. New York: Academic Press, 1975. Pp. 655-668.

Pomerantz, J. R., \& Garner, W. R. Stimulus configuration in selective attention tasks. Perception \& Psychophysics, 1973, 14, 565-569.

Saraga, E., \& Shallice, T. Parallel processing of the attributes of single stimuli. Perception \& Psychophysics, 1973, 13, 261-270.

Silverman, W. P., \& Goldaerg, S. L. Further confirmation of same versus different processing differences. Perception \& Psychophysics, 1975, 17, 189-193.

SNODGRASs, J. G. Reaction times for comparisons of successively presented visual patterns: Evidence for serial self-terminating search. Perception \& Psychophysics, 1972, 12, 364-372.

TVERSKY, B. Pictorial and verbal encoding in a short-term memory task. Perception \& Psychophysics, 1969, 6, 225-233.

WELL, A. D. The influence of irrelevant information on speeded classification tasks. Perception \& Psychophysics, 1971, 10, 79-84.

\section{NOTE}

1. The duration of the flash was about $40 \mathrm{msec}$ with rise and decay times within a $2-\mathrm{msec}$ limit. At a distance of $50 \mathrm{~cm}$ from the display, the intensity value was $0.54 \mu \mathrm{lm}$ per $\mathrm{cm}^{2}$ with a background illumination of $0.18 \mu \mathrm{lm}$ per $\mathrm{cm}^{2}$.

(Received for publication November 29, 1976; revision accepted January 20, 1977.) 